\title{
Diamond machining of freeform-patterned surfaces on precision rollers
}

\author{
Mingyu Liu ${ }^{1,2}$, Chi Fai Cheung ${ }^{1 *}$, Xiaobing Feng ${ }^{3}$ and Chunjin Wang ${ }^{1}$ \\ ${ }^{1}$ State Key Laboratory of Ultra-precision Machining Technology, Department of Industrial and Systems Engineering, The Hong \\ Kong Polytechnic University, Kowloon, Hong Kong \\ ${ }^{2}$ Manufacturing Metrology Team, Faculty of Engineering, University of Nottingham, Nottingham, United Kingdom \\ ${ }^{3}$ School of Mechanical Engineering, Shanghai Jiao Tong University, Shanghai, China \\ *Corresponding author: benny.cheung@ @olyu.edu.hk Tel: (852)27667905 Fax: (852)2362 5267
}

\begin{abstract}
:
Rapid development of freeform surfaces faces the challenges of not only higher form accuracy and smoother surface finishing, but also high machining efficiency and lower manufacturing cost. Combining diamond turning and roll-toroll embossing technologies is a promising solution to fulfil these requirements. This paper presents a generic method to design and machine freeform surfaces on precision rollers. The freeform surface designed on the flat substrate is first transferred onto the cylindrical roller surface. The freeform-patterned roller surface is then diamond turned using the toolpath generated by a purposely developed toolpath generator. With the proposed method, the complex freeform surfaces designed on flat substrate can be transferred to and precisely machined on the cylindrical roller surfaces. A cutting experiment has been conducted to demonstrate the capability of the proposed method. In the experiment, a sinusoidal surface was designed and diamond turned on a precision roller. The results demonstrate that the proposed method is accurate and effective. The proposed method provides guidance for the design and precision manufacturing of freeform-patterned surfaces on precision rollers.
\end{abstract}

Keywords: Ultra-precision machining, Freeform patterned surfaces, Diamond turning, Roll-to-roll, Precision surface measurement

\section{Introduction}

Precision freeform surfaces [1] and structured surfaces [2] are widely used in various consumer products such as digital cameras [3] and 3D displays [4], and applications in advanced optics [5] and bio-medical parts [6]. Future needs for these surfaces are not only high accuracy and fine surface finishing, but also high-volume and low-cost manufacturing. However, direct manufacturing of these kinds of surfaces using precision machining technologies such as lithography [7], single point diamond turning [8], and ion beam machining [9], etc., are usually costly and timeconsuming. Roll-to-roll (R2R) technology [10] is one promising technology to enable the cost- and time-effective production of freeform and structured surfaces, having successfully mass-produced components in LCD backlit panels [4], microlens arrays [11] and cylindrical lenses [12]. Manufacturing of the precision rollers, a key component of the R2R system, is critical for the R2R industry. Precision machining of such rollers has been demonstrated using beam pen lithography [13], combining of cylindrical photolithography, isotropic chemical etching, electropolishing methods [14], femtosecond laser exposure and the chemical wet-etching process [15], and the UV micro-stamping method [11]. However, most of these methods require expensive and complex manufacturing processes. There is a trend to machine freeform and structured patterns onto precision rollers using mechanical methods such as precision turning [16] and milling [17], which benefit from relatively simpler processes and lower cost.

Single point diamond turning is a promising technology which is capable of achieving high form accuracy and fine surface finishing. While most of the studies are focused on face turning [18-21], machining of precision rollers has received relatively little attention. Moreover, most studies on precision roller machining have applied taskspecified methods to machine specific types of surfaces. Lu et al. [22] developed a fast tool servo system to machine sinusoidal grid surface on a roller, where the sinusoidal grid was machined by simply correlating the motion of the fast tool servo with the rotation of the roller and the motion of the $z$ axis. The accuracy of the machined surface was difficult to ensure without precise synchronization of the two motions. Kong et al. [23] machined a wavy microstructured pattern on precision rollers using a single point diamond turning machine. Two-dimensional sinusoidal patterns with wavy cycles were machined on the roller with the proposed method. However, this method is only suitable for machining $2 \mathrm{D}$ sinusoidal patterns. It is difficult to apply this method to machine 3D freeform patterns on 
precision rollers. Li et al. [24] machined a V-shaped cylindrical grating on the roller surface, where the diamond tool cut in the $z$ axis direction while the workpiece rotated incrementally. This method is also limited to 2D patterns and cannot be directly used to machine 3D-patterned surfaces. With the help of an additional B-axis, Huang et al. [25] machined a radial Fresnel lens on precision rollers using diamond turning technology. However, the proposed method had very low efficiency. With an additional A-axis, Zhang et al. [26] improved the efficiency by proposing a swingingrotating diamond shaping (SDS) process. The method applies a specific design of the Fresnel lens on precision rollers. Huang et al. [27] proposed a diamond turning method to machine a microlens array on rollers, where the coordinates of the cutting tool tip were calculated for every single lenslet with a minimum distance method. On the whole, most of the above research studies are limited to specific tasks and the developed methods are difficult to be applied to freeform patterned surfaces with arbitrary designs, which require the capability to generate generic toolpaths.

While single point diamond turning is promising for the manufacture of freeform and micro-structured patterns on precision rollers, there are relatively few studies that focus on the development of a generic machining method for diamond turning of these kinds of freeform-patterned surfaces, i.e., given a designed surface with shape geometry of any degree of complexity, it can be transferred to the precision rollers, or the freeform patterns can be directly designed on the precision rollers, and be diamond machined as long as the tool geometry can meet the requirement. To fill this research gap, this paper presents a generic method starting from surface design to toolpath generation for diamond turning of freeform-patterned surfaces on precision rollers. Given a designed freeform surface with an arbitrary shape based on a flat substrate, the associated design on the roller surface can be determined and the toolpath can be generated using the purposely developed toolpath generator. The method is generic and can be used to machine complex freeform surfaces on the rollers. The details of the method are presented, and an experiment was conducted. The results demonstrate the effectiveness of the proposed method. The influences of the tool geometries are also discussed.

\section{Diamond Machining of Freeform Patterned Rollers}

The generic method for diamond machining of freeform surfaces on precision rollers consists of two parts. The first part is the design of freeform surfaces on rollers and the second part is toolpath generation. In most applications in the R2R industry, functional freeform surfaces are designed based on a flat substrate; hence, the first part aims to transfer the flat substrate-based freeform surfaces onto the cylindrical roller substrate. It should also be noted that the freeform patterned roller can also be designed directly based on the cylindrical surface. The roller substrate is then diamond turned with a helix-shaped toolpath, and a toolpath generator is purposely developed to generate the multi-axis motion for the precision diamond turning machine tool. The details of the method are given in the following sections.

\subsection{Design of freeform patterned surfaces on rollers}

Generally, the functional freeform surfaces are designed on a flat substrate using cartesian coordinates. The design of the freeform-patterned roller surface is used to transfer the freeform surfaces onto the cylindrical roller surface using cylindrical coordinates, as shown in Fig. 1.
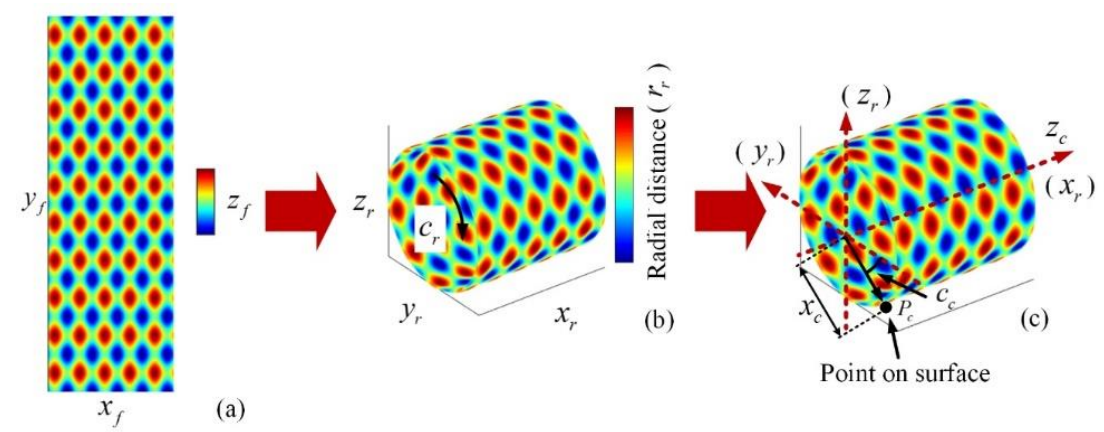

Fig. 1. Design of freeform surfaces on rollers, (a) freeform surface on flat substrate, (b) transferred to a cylindrical roller surface, and (c) transferred to the cylindrical coordinates of the machine tool

Fig. 1(a) shows the designed freeform pattern on a flat substrate in the $x_{f}-y_{f}-z_{f}$ coordination system, where the colormap represents the height in the $\mathrm{z}$ direction. The designed freeform pattern is then transferred onto a roller substrate as shown in Fig. 1(b), in the $x_{r}-c_{r}-r_{r}$ (also $x_{r}-y_{r}-z_{r}$ ) coordination system, where the lateral direction ( $\left.x_{f}\right)$, vertical direction $\left(y_{f}\right)$ and height direction $\left(z_{f}\right)$ as shown in Fig. 1(a) are transferred to the length direction $\left(x_{r}\right.$ 
), circumferential direction $\left(c_{r}\right)$ and radial distance $\left(r_{r}\right)$ (colormap) of the roller in Fig. 1(b), respectively. Ideally, the circumferential length of the cylinder (in Fig. 1(b)) should be equal to the length in the $y_{f}$ direction (in Fig. 1(a)). Hence, the radius of the roller $R_{r}$ is determined by Eq. (1).

$$
R_{r}=L_{y_{f}} / 2 \pi
$$

where $L_{y_{f}}$ is the length of the designed freeform surface in the $y_{f}$ direction.

Given any point $P$ on the surface, the relationship of its coordinates $\left(x_{r P}, c_{r P}, r_{r P}\right)$ in the cylindrical coordinate system $\left(x_{r}-c_{r}-r_{r}\right)$ and its coordinates $\left(x_{f P}, y_{f P}, z_{f P}\right)$ in the Cartesian coordinate system $\left(x_{f}-y_{f}-z_{f}\right)$ are determined by Eq. (2).

$$
\left\{\begin{array}{c}
x_{r P}=x_{f P} \\
r_{r P}=R_{r}+z_{f P} \\
c_{r P}=180 y_{f P} / \pi R_{r}
\end{array}\right.
$$

where $R_{r}$ is the designed radius of the roller. The transferred point on the roller surface can also be represented by the Cartesian coordinate system $x_{r}-y_{r}-z_{r}$ and its coordinates $y_{r P}$ and $z_{r P}$ can be determined by Eq. (3).

$$
\left\{\begin{array}{l}
y_{r P}=r_{r P} \cos \left(c_{r P}\right) \\
z_{r P}=r_{r P} \sin \left(c_{r P}\right)
\end{array}\right.
$$

To be consistent with the coordinates of the diamond turning machine, the data of the surface is then transferred to the format as shown in Fig. 1(c), which is in the cylindrical coordinate system $\left(z_{c}-x_{c}-c_{c}\right)$ along the cylindrical axis $\left(z_{c}\right)$, radial distance $\left(x_{c}\right)$ and azimuth $\left(c_{c}\right)$ according to the axes of the machine tool, i.e. $z, x$ and $c$ axes, respectively. Hence, the same surface point on the roller can be represented in the $z_{c}-x_{c}-c_{c}$ coordinate system, and is determined by:

$$
\left\{\begin{array}{l}
z_{c P}=x_{r P} \\
x_{c P}=r_{r P} \\
c_{c P}=c_{r P}
\end{array}\right.
$$

In the above calculation, a larger $R_{r}$ is possible which results in a gap in the roller surface, which can be filled with additional data. However, a smaller $R_{r}$ needs to be avoided since it introduces an overlap between parts of the designed surface. When the flat substrate is designed to be transferred to the cylindrical substrate, special attention should be paid to avoid surface discontinuity in the wrap-around region, which may affect the dynamics of the machining process.

\subsection{Toolpath generation}

After the designed surface is transferred into the representation format associated with the motion axes of the machine tool, the locus of the diamond tool can then be determined using the toolpath generator described as follows. The toolpath generator is purposely built to determine the motion trajectory of the centre of the diamond tool, where the trajectory is generated in a helix shape along the freeform-patterned cylindrical surface. Motion along the trajectory is determined by keeping the diamond tool oriented tangent to the roller surface at every sampling position, as shown in Fig. 2 (the helix is shown in a large pitch for better visualization). As shown in Fig. 1(b), the workpiece rotation is equivalent to the cutting plane of the diamond tool rotating in the reverse direction shown in Fig. 2(a). It is assumed that the cutting edge of the diamond tool is a perfect circle, so the centre locations of the diamond tool in each cutting plane can be determined by offsetting the designed surface profile on the roller by a distance of $r$, where $r$ is the radius of the cutting edge of the diamond tool, as shown in Fig. 2(b).

In the turning process, the workpiece is rotated by the spindle in the $c$ direction and the diamond tool feeds in the $z$ direction, together with the location of cutting determined in the $x$ direction. Hence, the turning process is a $c-z-x 3-$ axes servo motion process. As the workpiece rotates around the $c$ axis, the actual coordinate of the diamond tool in the $z$ axis $z_{A}$ is determined by:

$$
z_{A}=P_{i t} c_{A} / 360
$$

where $P_{i t}$ is the pitch of the helix and $c_{A}$ is the actual angle of the workpiece (spindle). 

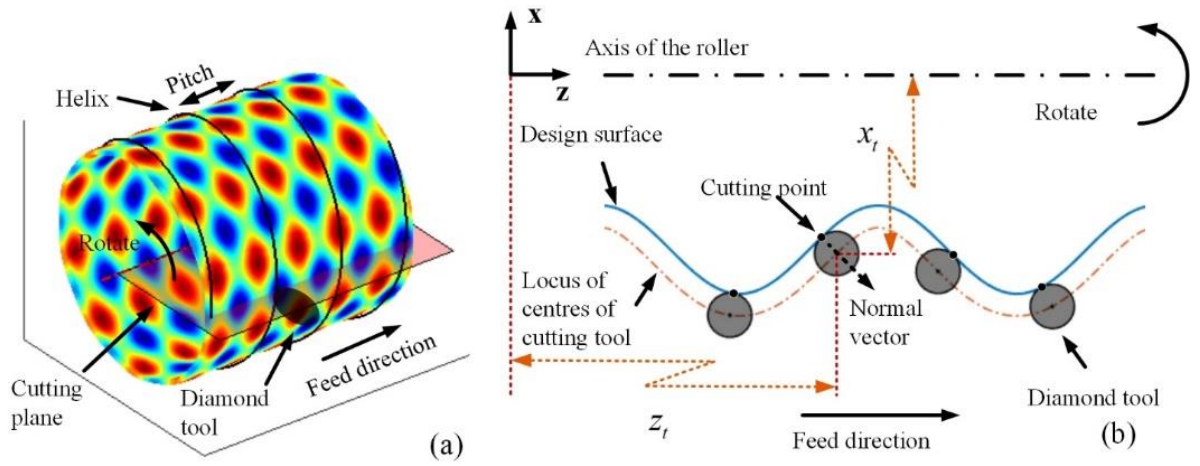

Fig. 2. Toolpath generation, (a) cutting process with helix toolpath (b) profile view at cutting plane

As the workpiece rotates, the cutting plane rotates in the reverse direction. The cross section of the freeformpatterned roller surface and the cutting plane can be determined as shown in Fig. 2(b). The cross section is a profile presented as discrete points in the $z_{c}-x_{c}$ plane, and it can be determined by the intersection data of the designed 3D roller surface and the cutting plane. Corresponding points of the offset profile in $z_{c}-x_{c}$ plane $\left(z_{t p}, x_{t p}\right)$ can then be determined by:

$$
\left\{\begin{array}{l}
z_{t p}=z_{c p}-r d_{x_{c p}} \\
x_{t p}=x_{c p}+r d_{z_{c p}}
\end{array}\right.
$$

where $r$ is the tool radius, $d_{x_{c p}}$ and $d_{z_{c p}}$ are the components in the $x_{c}$ and $z_{c}$ directions for the unit normal vector at every point on the profile, respectively. $d_{x_{c p}}$ and $d_{z_{c p}}$ can be determined by:

$$
\left\{\begin{array}{l}
d_{x_{c p}}=G_{x_{c p}} \bullet f_{n} \\
d_{z_{c p}}=G_{z_{c p}} \bullet f_{n}
\end{array}\right.
$$

where $G_{x_{c p}}$ and $G_{z_{c p}}$ are the numerical gradients of the profile in the $x$ and $z$ directions, respectively. $f_{n}$ is the normalized factor. The gradients can be determined by:

$$
\begin{aligned}
& \left\{\begin{array}{l}
G_{x_{c p}}=\partial f_{x} / \partial x_{c p} \\
G_{z_{c p}}=\partial f_{z} / \partial z_{c p}
\end{array}\right. \\
& f_{n}=\frac{1}{\sqrt{G_{x}^{2}+G_{z}^{2}}}
\end{aligned}
$$

Finally, the actual position of the tool centre in the $x$ direction is determined by interpolating the offset profile.

\section{Experiment verification and discussion}

\subsection{Roller with sinusoidal patterns}

To evaluate the effectiveness of the proposed method, a freeform patterned surface was designed, as shown in Fig. 3. The freeform patterned surface is defined by:

$$
z=0.02[\sin (5 x)+\cos (10 y / 3)]-0.04
$$

where $x \in[0,6.28]([0,2 \pi]) \mathrm{mm}$ and $y \in[0,18.84]([0,6 \pi]) \mathrm{mm}$. The substrate is the flat surface on the $x-y$ plane with $z=0$.

The freeform patterned surface is then transferred to the roller surface with a radius of $R$, where $R=6 \pi / 2 \pi=3$ $\mathrm{mm}$. In this design, the number of periods of sinusoidal patterns in the $y$ direction (in Fig. 3) is 10. As a result, it forms a continuous sinusoidal pattern on the cylindrical surface as shown in Fig. 4, where the colormap represents the radial distance (RD). 


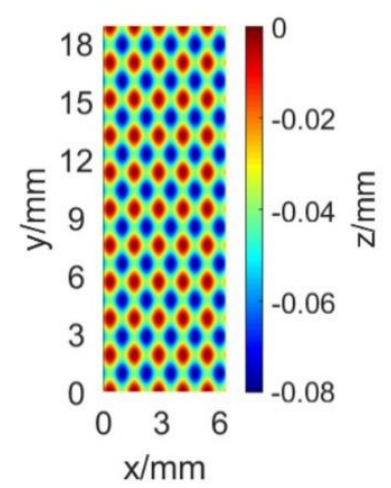

Fig. 3. Designed sinusoidal patterns on a flat substrate

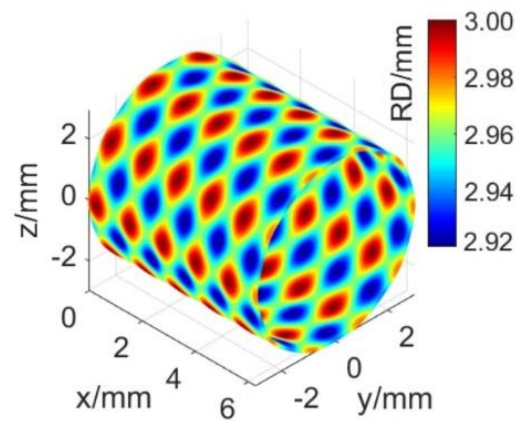

Fig. 4. Sinusoidal patterned roller surface

The material of the workpiece in this experiment was brass. The rough blank was prepared to have a slightly larger radius of $3.01 \mathrm{~mm}$. The rough blank was first machined to have a smooth surface and then followed by the slow tool servo (STS) turning. While the designed surface had the length of $6.28 \mathrm{~mm}$ in the $x$ direction, the machining area could be chosen to be a shorter length, and it was chosen to be $5.5 \mathrm{~mm}$ in this experiment. The STS turning consists of rough and fine machining processes. In rough turning, the step resolution of the $c$ axis was $1^{\circ}$ and the pitch for every revolution was $0.1 \mathrm{~mm}$. In fine turning, the step resolution of the $c$ axis was $0.1^{\circ}$ and the pitch of the helix was 0.005 $\mathrm{mm}$. For each machining cycle, the $x$ axis of the machine tool was offset by a distance equal to the depth of cut. The material was then removed layer by layer to obtain the designed radius of $3 \mathrm{~mm}$. The tool radius of the diamond tool used in the experiment was determined to be $1.417567 \mathrm{~mm}$. The machine tool was a Moore Nanotech 450FG ultraprecision machine. The machining parameters are summarised in Table 1.

Table 1 Machining parameters of the freeform patterned roller

\begin{tabular}{lll}
\hline & Rough machining & Fine machining \\
\hline Machine tool & Moore Nanotech 450FG & \\
Workpiece material & Brass & \\
Resolution of $c$ axis & $1^{\circ}$ & $0.1^{\circ}$ \\
Pitch & $0.1 \mathrm{~mm}$ & $0.005 \mathrm{~mm}$ \\
Cutting length in $z$ direction & $5.5 \mathrm{~mm}$ & \\
Tool material & Single crystal diamond & \\
Tool radius & $1.417567 \mathrm{~mm}$ & \\
Tool rake angle & $0^{\circ}$ & \\
Tool clearance angle & $12.5^{\circ}$ & \\
Tool opening angle & $100^{\circ}$ & \\
\hline
\end{tabular}


With regard to rough machining, for example, the characteristics of the toolpath is shown in Fig. 5. During the machining process, the diamond tool moves from $0.5 \mathrm{~mm}$ to $6 \mathrm{~mm}$ in the $z$ direction, corresponding to the $5.5 \mathrm{~mm}$ cutting length. The angles in the $\mathrm{c}$ axis ranged from $0-359^{\circ}$ in every revolution. The $x$ axis motion adapts to the freeform-structured pattern of the roller and thus is the most heavily-loaded axis, as shown in Fig. 5. Fig. 6 shows the toolpath around the workpiece.

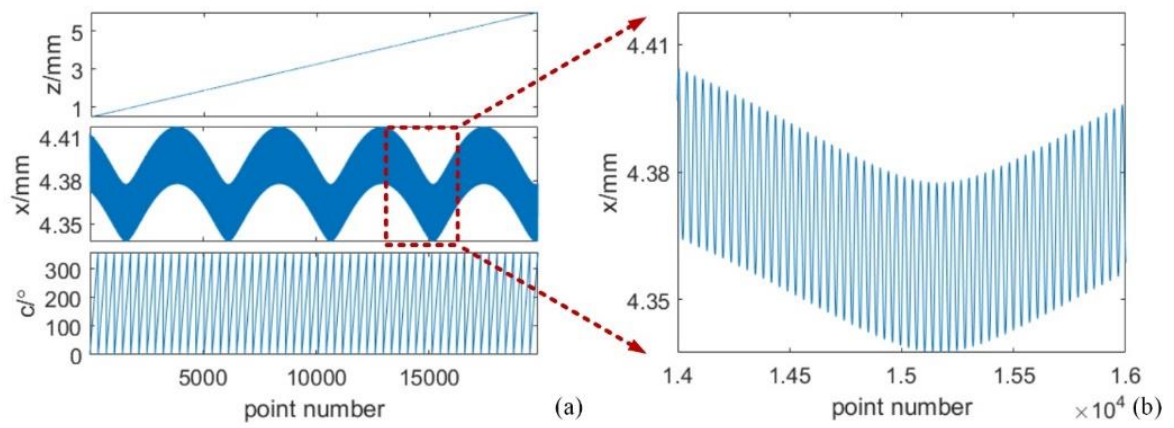

Fig. 5. Characteristics of the toolpath generated, (a) for motions of $\mathrm{z}, \mathrm{x}, \mathrm{c}$ axes, and (b) enlarged view of motion of the $\mathrm{x}$ axis
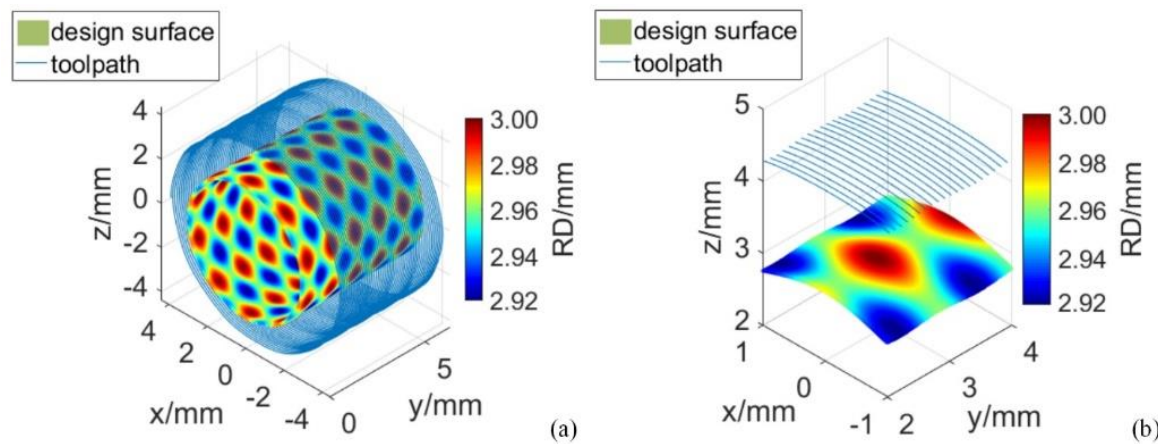

Fig. 6. 3D views of the designed surface and toolpath (for rough machining), (a) the overall view, and (b) enlarged view

The machining process is shown in Fig. 7(a). The machined workpiece was measured by a Zygo NexView coherence scanning interferometer (CSI) with a 20x objective, as shown in Fig. 7(b). An area of approximately $4 \mathrm{~mm}$ $\times 0.8 \mathrm{~mm}$ along the cylindrical surface was measured and the measurement result is shown in Fig. 8. A cross sectional profile of the surface is also shown in Fig. 8. The result shows a perfect sinusoidal shape and the enlarged view shows that the surface is smooth. The surface roughness was also analysed. Fig. 9 shows the surface micro-topography after form removal. The result shows that the surface roughness $S_{a}$ was $7 \mathrm{~nm}$. The measured surface was further evaluated quantitatively by matching the designed surface with the measured surface using the iterative closest point (ICP) method [28] as shown in Fig. 10. Fig. 10(a) shows the registration result and Fig. 10(b) shows the error map. The rootmean-square (RMS) value and the peak-to-valley (PV) value of the error map were $0.267 \mu \mathrm{m}$ and $4.9 \mu \mathrm{m}$, respectively. A relatively large PV value was caused by pits and chips on the surface, as shown in the enlarged views in Fig. 10(b). On the whole, the machined workpiece had sub-micrometre form accuracy.

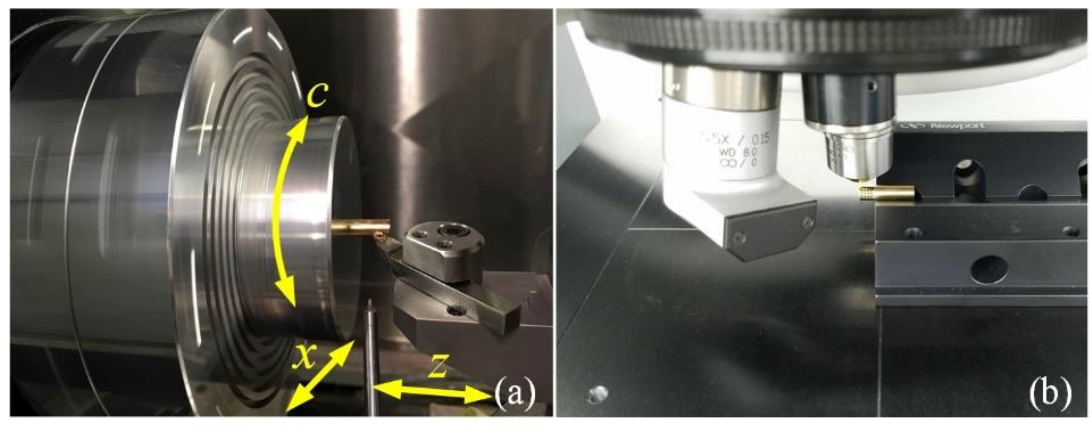

Fig. 7. Snapshots of (a) diamond turning process, and (b) measurement process 

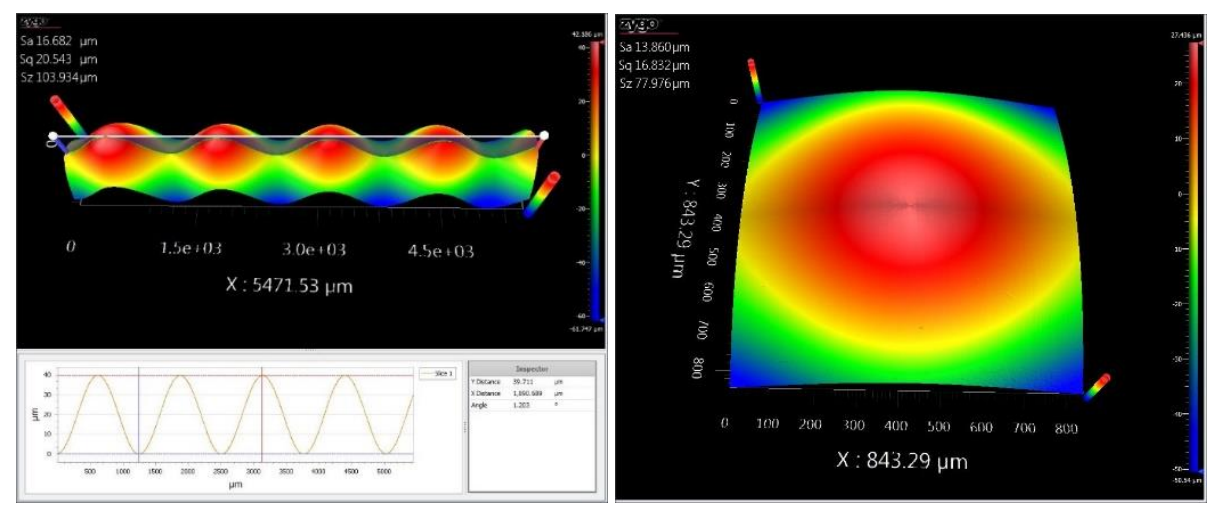

Fig. 8. Measurement results of the machined workpiece

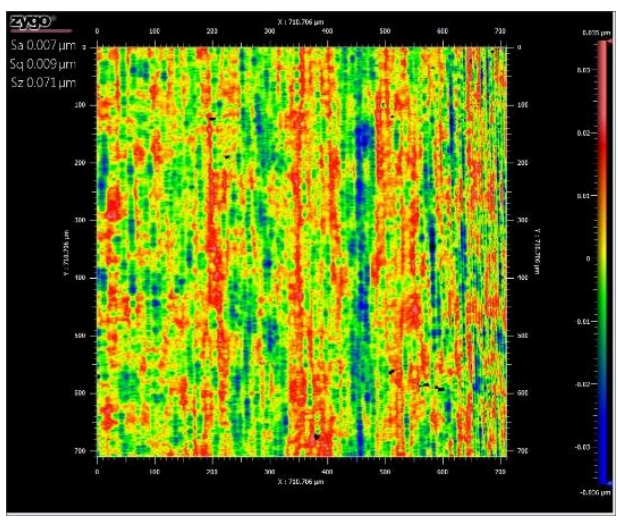

Fig. 9. Surface micro-topography after form removal
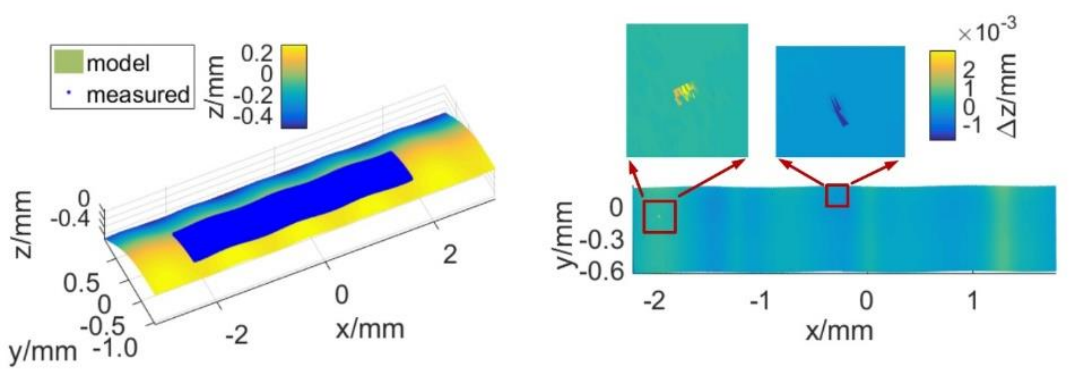

(a)

(b)

Fig. 10. Characterization result of the measured surface, (a) registration result, and (b) error map

\subsection{Roller with a microlens array}

Another experiment was conducted to fabricate a microlens array on a precision roller. The microlens array was designed with rectangular apertures to increase the fill factor [29,30]. In this experiment, the microlens array was directly designed on the roller instead of designing the microstructure patterns on a flat surface. The microlenses were designed as ellipsoids and the surface was determined by:

$$
\frac{x^{2}}{a^{2}}+\frac{y^{2}}{b^{2}}+\frac{z^{2}}{c^{2}}=1
$$

where $a=b=0.505 \mathrm{~mm}$ and $c=1.01 \mathrm{~mm}$ are the length of the principal semi-axes of the ellipsoid. The original radius of the roller was designed to be $3 \mathrm{~mm}$ and the ellipsoid was imprinted on the roller surface in the lateral direction along the cylindrical axis with a distance of $100 \mu \mathrm{m}$ from the roller surface and in the circumference direction along the roller surface with a $3.75^{\circ}$ angular distance, i.e. the microlenses overlapped and resulted in rectangular apertures of approximately $100 \mu \mathrm{m} \times 200 \mu \mathrm{m}$, while the depth of the microlens was approximately $5 \mu \mathrm{m}$. The designed microlens array-patterned roller is shown in Fig. 11. 


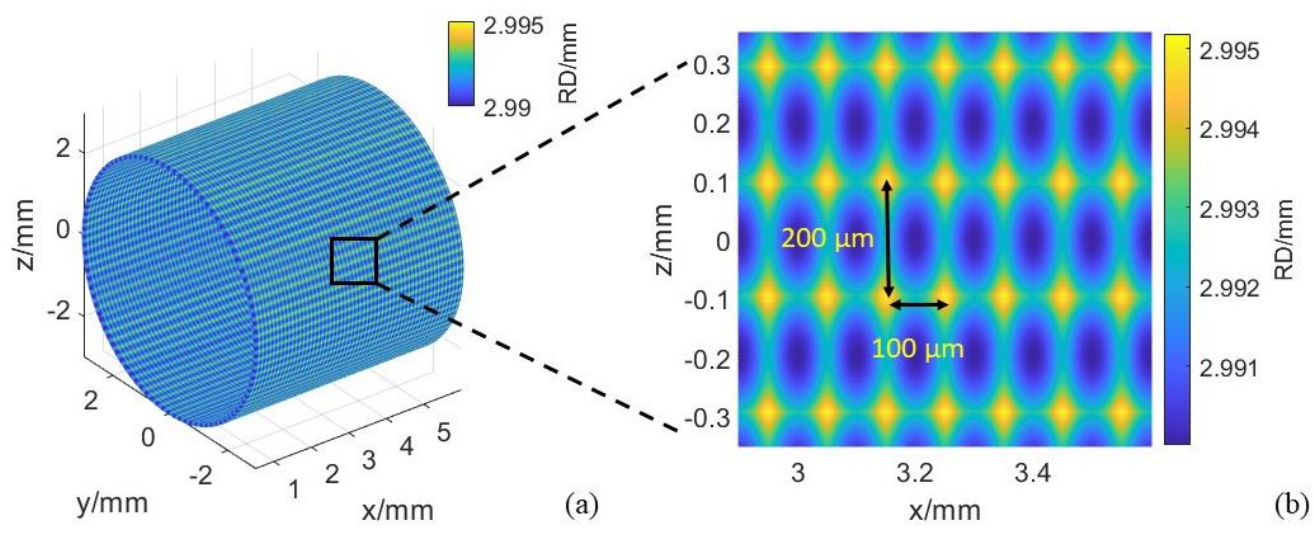

Fig. 11. Design of microlens array on a precision roller, (a) the overall view, and (b) enlarged view

The workpiece was machined by a Moore Nanotech 450FG diamond turning machine tool and the material was brass. A single crystal diamond tool with a tool radius of $0.074239 \mathrm{~mm}$ was used. The machined workpiece was measured using an optical microscope. The microlens array with rectangular apertures of $100 \mu \mathrm{m} \times 200 \mu \mathrm{m}$ is clearly shown in Fig. 12(a) and the tool mark with a 4- $\mu \mathrm{m}$ pitch distance is shown in Fig. 12(b).
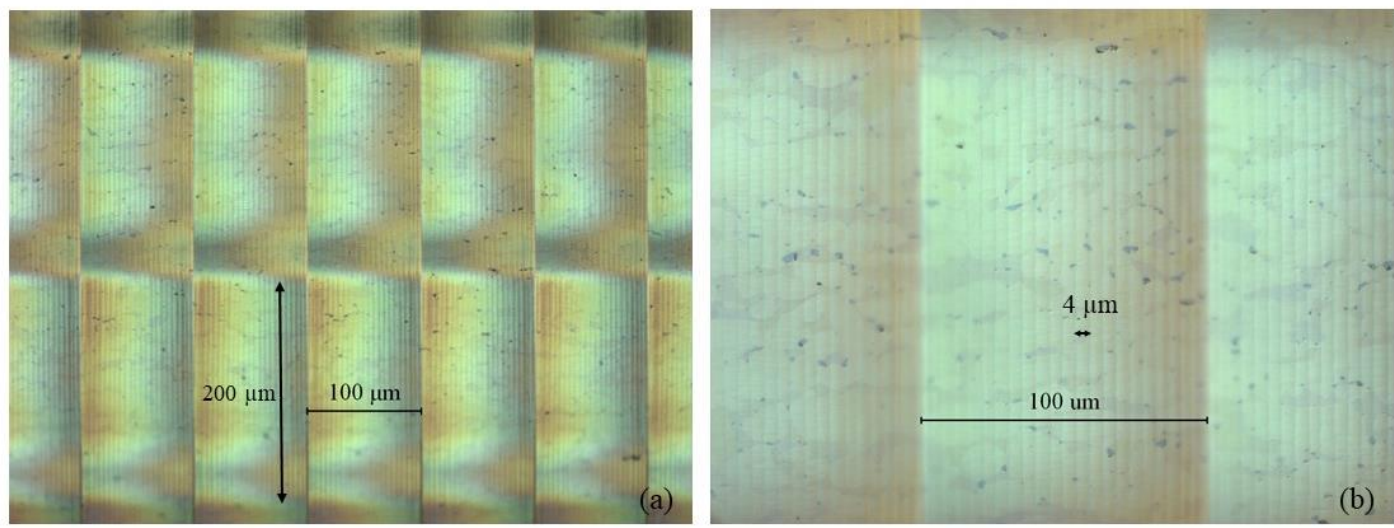

Fig. 12. Machined workpiece measured by an optical microscope, (a) roller surface of the microlens array with rectangular apertures, and (b) enlarged view showing tool marks

This experiment demonstrates that the method is capable of machining freeform patterns with sharp edges on the precision rollers, as well as continuous freeform patterned surfaces such as that shown in section 3.1. It is noted that, the limitation of the method is that it cannot machine surfaces with sharp corners as the proposed method is designed for diamond tools with circular geometries. To machine those surfaces with sharp corners, special tools with a small tool nose radius should be used.

\section{Discussion of the constraints of tool geometries}

As the diamond tool is assumed to have circular geometry, the proposed surface design and toolpath generation method can be used to design and manufacture complex surfaces on rollers, as long as the following restrictions caused by tool geometries are taken into consideration:

1. Tool radius. The tool radius affects the minimum radius of curvature of the machined surface, especially in the cylindrical axis direction. The minimum radius of curvature (MR) should be determined when the surface is designed. The tool radius needs to be smaller than MR, as shown in Fig. 13. 


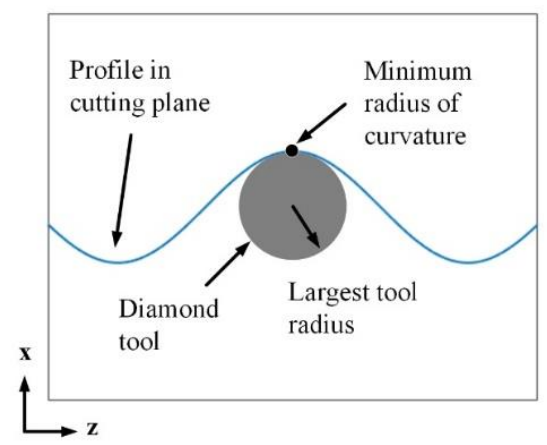

Fig. 13. Relationship of tool radius and radius of curvature of the machined surface in the cutting plane

The radius of curvature $R_{c}$ is determined by:

$$
R_{c}=\left|\frac{\left(1+x^{\prime 2}\right)^{\frac{3}{2}}}{x^{\prime \prime}}\right|
$$

where $x^{\prime}=\frac{d x}{d z}$ and $x^{\prime \prime}=\frac{d^{2} x}{d z^{2}}$ are the first and the second derivatives of the function of the profile in the cutting plane.

With regard to the first experiment, for example, the minimum radius of curvature of the freeform surface was determined to be $2 \mathrm{~mm}$ and the tool radius was chosen to be $1.417567 \mathrm{~mm}$.

2. Tool clearance angle. The tool clearance angle affects the maximum surface slope of the machined surface, in the circumferential direction. The tool clearance angle must be larger than the maximum surface slope in the cutting direction, i.e. $\beta>\alpha$, as shown in Fig. 14. For example, in the first experiment, the maximum surface slope of the workpiece in the cutting direction was determined to be $3.81^{\circ}$, and the clearance angle of the diamond tool was $12.5^{\circ}$, which was well above the maximum surface slope of the workpiece in the cutting direction.

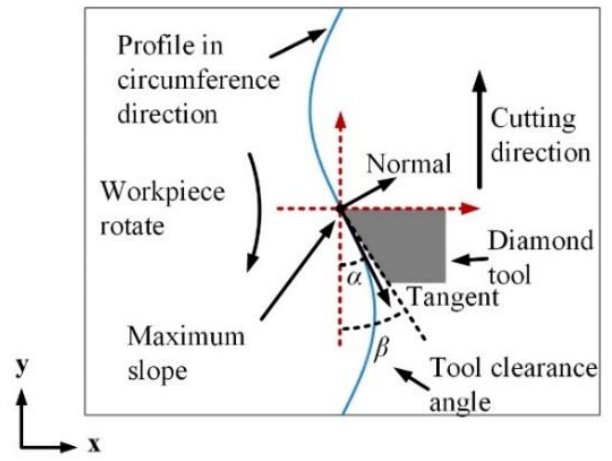

Fig. 14. Relationship of the tool clearance angle and the surface slope in the cutting direction

3. Tool opening angle. The toolpath generator assumes that the cutting edge of the diamond tool is circular. However, the tool has a limited opening angle and this affects the maximum slope of the surface in the cylindrical axis direction, as shown in Fig. 15. In the first experiment, the maximum surface slope $\theta$ " for the machined workpiece was determined to be $5.71^{\circ}$. The opening angle $\theta$ for the diamond tool was $100^{\circ}$. The maximum acceptable surface slope $\theta^{\prime}$ was determined to be $\theta / 2=50^{\circ}$, which was well above the maximum surface slope of the workpiece. 


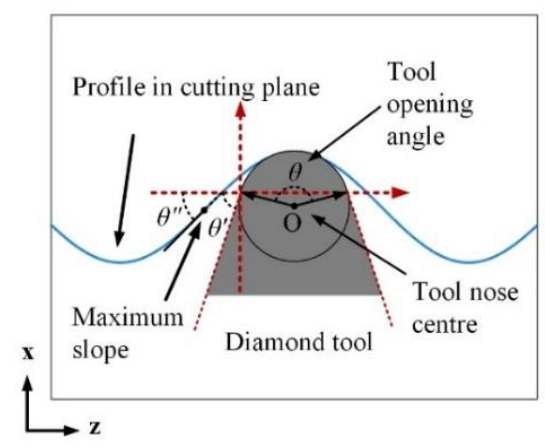

Fig. 15. Relationship of opening angle of the cutting tool and surface slope in the cutting plane

It is noted that if the designed surface and the diamond tool cannot meet the requirements, either the designed surface or the diamond tool needs to be modified to meet the geometrical requirement. Diamond tools with sharp edge geometries were not within the scope of this study.

\section{Conclusion}

A generic method to design and machine freeform patterned surfaces on precision rollers is presented. The method first transfers the conventional freeform surface designed on the flat substrate to the cylindrical roller surface. Alternatively, the freeform patterns can be directly designed on the precision roller. A toolpath generator is developed to machine the freeform-patterned roller surfaces. Experimental results prove that the proposed method is accurate and effective. Influences of tool geometries are also discussed. The limitation of the proposed method is that the design of the toolpath generator is based on circular diamond tools rather than sharp diamond tools. Hence, it is difficult to generate toolpaths for surfaces with sharp corners. However, it is possible to machine surfaces with sharp convex edges. This limitation can be considered as part of the influence of the tool geometries. Due to the generic nature of the proposed method, it is expected to be widely applicable in the roll-to-roll industry, to machine freeform and structure-patterned precision rollers.

\section{Acknowledgments}

The work described in this paper was mainly supported by a grant from the Ministry of Science and Technology of China (Project Code: 2017YFE0191300). The authors would also like to express their thanks to the Research Office of The Hong Kong Polytechnic University (Project code: BBX7). Many thanks are also due to Mr. Tai Wa Chung and Mr. Tsz Hin Fan for their help in the experiments.

\section{References}

1. Ries H, Muschaweck J (2002) Tailored freeform optical surfaces. J Opt Soc Am A 19 (3):590-595

2. Evans CJ, Bryan JB (1999) "Structured","textured" or "engineered" surfaces. CIRP Annals - Manufacturing Technology 48 (2):541-556

3. Song YM, Xie Y, Malyarchuk V, Xiao J, Jung I, Choi K-J, Liu Z, Park H, Lu C, Kim R-H, Li R, Crozier KB, Huang Y, Rogers JA (2013) Digital cameras with designs inspired by the arthropod eye. Nature 497:95

4. Fattal D, Peng Z, Tran T, Vo S, Fiorentino M, Brug J, Beausoleil RG (2013) A multi-directional backlight for a wide-angle, glasses-free threedimensional display. Nature 495 (7441):348

5. Fang FZ, Zhang XD, Weckenmann A, Zhang GX, Evans C (2013) Manufacturing and measurement of freeform optics. CIRP Annals Manufacturing Technology 62 (2):823-846

6. Giannatsis J, Dedoussis V (2009) Additive fabrication technologies applied to medicine and health care: a review. The International Journal of Advanced Manufacturing Technology 40 (1):116-127

7. Chou SY, Krauss PR, Renstrom PJ (1996) Imprint Lithography with 25-Nanometer Resolution. Science 272 (5258):85

8. Yi AY, Li L (2005) Design and fabrication of a microlens array by use of a slow tool servo. Opt Lett 30 (13):1707-1709

9. Tseng AA (2004) Recent developments in micromilling using focused ion beam technology. Journal of Micromechanics and Microengineering $14(4): R 15$

10. Bae S, Kim H, Lee Y, Xu X, Park J-S, Zheng Y, Balakrishnan J, Lei T, Ri Kim H, Song YI, Kim Y-J, Kim KS, Ozyilmaz B, Ahn J-H, Hong BH, Iijima S (2010) Roll-to-roll production of 30-inch graphene films for transparent electrodes. Nat Nano 5 (8):574-578

11. Yang S-Y, Cheng F-S, Xu S-W, Huang P-H, Huang T-C (2008) Fabrication of microlens arrays using UV micro-stamping with soft roller and gas-pressurized platform. Microelectronic engineering 85 (3):603-609

12. Shan X, Liu T, Mohaime M, Salam B, Liu Y (2015) Large format cylindrical lens films formed by roll-to-roll ultraviolet embossing and applications as diffusion films. Journal of Micromechanics and Microengineering 25 (3):035029

13. Tsai S-W, Chen P-Y, Lee Y-C (2014) Fabrication of a seamless roller mold with wavy microstructures using mask-less curved surface beam pen lithography. Journal of Micromechanics and Microengineering 24 (4):045022 
14. Lee YC, Chen HW, Hsiao FB (2012) Fabrication of Seamless Roller Mold for Continuous Roller Imprinting of Microlens Array Films. Journal of Microelectromechanical Systems 21 (2):316-323

15. Du G, Yang Q, Chen F, Liu H, Deng Z, Bian H, He S, Si J, Meng X, Hou X (2012) Direct fabrication of seamless roller molds with gapless and shaped-controlled concave microlens arrays. Opt Lett 37 (21):4404-4406

16. Cheung C, Lee W (2000) A theoretical and experimental investigation of surface roughness formation in ultra-precision diamond turning. International Journal of Machine Tools and Manufacture 40 (7):979-1002

17. Cheung C, Kong L, Lee W, To S (2006) Modelling and simulation of freeform surface generation in ultra-precision raster milling. Proceedings of the Institution of Mechanical Engineers, Part B: Journal of Engineering Manufacture 220 (11):1787-1801

18. Fang F, Zhang X, Hu X (2008) Cylindrical coordinate machining of optical freeform surfaces. Optics Express 16 (10):7323-7329

19. Li L, Yi AY (2010) Development of a 3D artificial compound eye. Optics Express 18 (17):18125-18137

20. Zhu Z, To S, Zhang S (2015) Large-scale fabrication of micro-lens array by novel end-fly-cutting-servo diamond machining. Optics Express $23(16): 20593-20604$

21. Kong L, Cheung C, To S, Wang B, Ho L (2014) A theoretical and experimental investigation of design and slow tool servo machining of freeform progressive addition lenses (PALs) for optometric applications. The International Journal of Advanced Manufacturing Technology 72 (14):33-40

22. Lu H, Lee D-W, Lee S-M, Park J-W (2012) Diamond machining of sinusoidal grid surface using fast tool servo system for fabrication of hydrophobic surface. Transactions of Nonferrous Metals Society of China 22:s787-s792

23. Kong LB, Cheung CF, Lee WB (2016) A theoretical and experimental investigation of orthogonal slow tool servo machining of wavy microstructured patterns on precision rollers. Precision Engineering 43:315-327

24. Li G, Xu Z, Fang F, Wu W, Xing X, Li W, Liu H (2013) Micro cutting of V-shaped cylindrical grating template for roller nano-imprint. Journal of Materials Processing Technology 213 (6):895-904

25. Huang R, Zhang XQ, Rahman M, Kumar AS, Liu K (2015) Ultra-precision machining of radial Fresnel lens on roller moulds. CIRP Annals Manufacturing Technology 64 (1):121-124

26. Zhang X, Huang R, Kumar AS, Liu K (2018) High-efficiency swinging-rotating diamond shaping of Fresnel lenses on roller molds. CIRP Annals - Manufacturing Technology

27. Huang P, To S, Zhu Z (2018) Diamond turning of micro-lens array on the roller featuring high aspect ratio. The International Journal of Advanced Manufacturing Technology 96 (5-8):2463-2469

28. Besl PJ, McKay ND (1992) A method for registration of 3-D shapes. IEEE Transactions on pattern analysis and machine intelligence 14 (2):239256

29. Pang K, Song L, Fang F, Zhang Y, Zhang H (2016) An imaging system with a large depth of field based on an overlapped micro-lens array. CIRP Annals 65 (1):471-474.

30. Cheung CF, Liu M, Leach R, Feng X, Zhao C (2018) Hierarchical-information-based characterization of multiscale structured surfaces. CIRP Annals 67 (1):539-542. 